\title{
Risk-stratified surveillance protocol improves cost-effectiveness after radical nephroureterectomy in patients with upper tract urothelial carcinoma
}

\author{
Masaki Momota ${ }^{1}$, Shingo Hatakeyama ${ }^{1}$, Hayato Yamamoto ${ }^{1}$, Hiromichi Iwamura ${ }^{1,2}$, \\ Yuki Tobisawa ${ }^{1}$, Tohru Yoneyama ${ }^{3}$, Takahiro Yoneyama ${ }^{1}$, Yasuhiro Hashimoto ${ }^{3}$, \\ Takuya Koie ${ }^{1}$, Ikuya Iwabuchi ${ }^{4}$, Masaru Ogasawara ${ }^{4}$, Toshiaki Kawaguchi ${ }^{4}$ and \\ Chikara Ohyama ${ }^{1,3}$ \\ ${ }^{1}$ Department of Urology, Hirosaki University Graduate School of Medicine, Hirosaki, Japan \\ ${ }^{2}$ Department of Urology, Tohoku Medical and Pharmaceutical University, Sendai, Japan \\ ${ }^{3}$ Department of Advanced Transplant and Regenerative Medicine, Hirosaki University Graduate School of Medicine, Hirosaki, \\ Japan \\ ${ }^{4}$ Department of Urology, Aomori Prefectural Central Hospital, Aomori, Japan \\ Correspondence to: Shingo Hatakeyama, email: shingoh@hirosaki-u.ac.jp \\ Keywords: upper tract urothelial carcinoma; radical nephroureterectomy; recurrence; surveillance; cost-effectiveness \\ Received: December 26, $2017 \quad$ Accepted: April 06, $2018 \quad$ Published: May 01, 2018
}

Copyright: Momota et al. This is an open-access article distributed under the terms of the Creative Commons Attribution License 3.0 (CC BY 3.0), which permits unrestricted use, distribution, and reproduction in any medium, provided the original author and source are credited.

\section{ABSTRACT}

Objectives: To develop a surveillance protocol with improved cost-effectiveness after radical nephroureterectomy (RNU), as the cost-effectiveness of oncological surveillance after RNU remains unclear.

Results: Of 426 patients, 109 (26\%) and $113(27 \%)$ experienced visceral and intravesical recurrences, respectively. The pathology-based protocol found significant differences in recurrence-free survival in the visceral recurrence but not in the intravesical recurrence. The medical costs per visceral recurrence detected were high, especially in normal-risk ( $\leq$ pT2N0, LVI-, SM-) patients. We developed a risk score associated with visceral recurrence using Cox regression analysis. The risk score-based protocol was significantly more cost-effective than the pathology-based protocol. Estimated cost differences reached $\$ 747,929$ per recurrence detected, a suggested $55 \%$ reduction.

Materials and Methods: We retrospectively evaluated 426 patients with RNU for upper tract urothelial carcinoma (UTUC) without distant metastasis at 4 hospitals. Patients with routine oncological follow-up were stratified into normal-, high- and very high-risk groups according to a pathology-based protocol utilizing pathological stage, lymphovascular invasion (LVI) and surgical margin (SM). Cost-effectiveness of the pathology-based protocol was evaluated, and a risk score-based protocol was developed to optimize cost-effectiveness. Risk scores were calculated by summing up risk factors independently associated with recurrence-free survival. Patients were stratified by low-, intermediate- and high-risk score. Estimated cost per recurrence detected by pathology-based and risk score-based protocols was compared.

Conclusions: A risk score-stratified surveillance protocol has the potential to reduce over investigation during follow-up, making surveillance more cost-effective.

\section{INTRODUCTION}

Upper tract urothelial carcinoma (UTUC) is relatively rare, and radical nephroureterectomy (RNU) with bladder cuff excision remains the standard treatment modality for non-metastatic UTUC [1]. The prognosis of patients with locally advanced UTUC has not improved over the past 2 decades [2-6] despite established predictors of prognosis in patients with UTUC including older age, tumor stage, presence of hydronephrosis, tumor 
location, lymphovascular invasion (LVI), surgical margin (SM) and lymph node involvement [3, 7-10]. In addition, the effective follow-up frequency and evaluation methods remain unclear, although various surveillance regimens have been proposed for muscle-invasive bladder cancer [11-15]. Furthermore, there is a lack of evidence regarding the cost-effectiveness of routine oncological follow-up to detect recurrence after RNU. Therefore, we aimed to develop the optimal surveillance protocol to improve cost-effectiveness after RNU. First, we estimated the per person cost of detecting post-RNU recurrence using our pathology-based protocol. Further, we developed a novel risk score-based protocol using Cox proportional hazards regression and compared the cost-effectiveness of the pathology- and risk score-based protocols.

\section{RESULTS}

\section{Background of patients}

Of 426 patients with RNU, 109 (26\%) and 113 (27\%) experienced visceral and intravesical recurrences, respectively. Of these, $209(49 \%), 182(43 \%)$, and 35 $(8.2 \%)$ were included in the pathology-based normalrisk, high-risk, and very high-risk group, respectively. The clinicopathological characteristics are shown in Table 1. Pathological outcomes ( $\geq$ pT3, LVI+, SM+) were significantly worse in high- and very high-risk patients compared to normal risk-patients. There were no significant differences in clinical parameters except for $\geq$ $\mathrm{cT} 3, \mathrm{cN}+$, and presence of hydronephrosis between high-/ very high-risk and normal risk-patients.

\section{Oncological and economic outcomes of pathology-based surveillance protocols}

Time course of intravesical and visceral recurrences in all patients was shown in Figure 1A. Most patients experienced visceral $(n=79 / 109 ; 72 \%)$ and intravesical $(n=87 / 113 ; 80 \%)$ recurrences within 24 months. Visceral recurrence-free survival in the 3 groups was also significantly different $(P<0.001$; Figure 1B). The number of patients with visceral recurrence within 24 months was significantly different among patients with normal-risk $(n=13 / 209 ; 6.2 \%)$, high-risk $(n=49 / 182 ; 27 \%)$, and very high-risk $(n=$ $17 / 35$; 49\%) $(P<0.001$; Figure $1 C)$. On the other hand, intravesical recurrence-free survival in the 3 groups was not significantly different (Figure 1D). The number of patients with intravesical recurrence within 24 months was not significantly different among patients with normal risk $(n=44 / 209 ; 21 \%)$, high risk $(n=38 / 182$; $27 \%)$, and very high risk $(n=5 / 35 ; 14 \%)(P=0.642$; Figure 1E). The estimated cost per detected recurrence was acceptable within 18 months in the normal-risk and high-risk group $(<\$ 50,000)$ but it increased thereafter
(Figure 1F). The cost per detected recurrence was very low in the very high-risk group for 5 years $(<\$ 10,000)$.

\section{Oncological and economic outcomes of risk score-based surveillance protocols}

Uni- and multivariate Cox regression analysis found 7 factors that were independently associated with recurrence-free survival, including $\mathrm{SM}+$ (score 2), LVI+ (score 2), $\geq \mathrm{pT} 3$ or 4 (score 2), preoperative stage $3 \mathrm{CKD}$ (score 2), $\mathrm{cN}+$ or $\mathrm{pN}+$ (score 2), hydronephrosis (score 1), and tumor location (score 1) (Table 2, Figure 2A). When hazard ratio was greater than 3 , the score was marked as 2 . The risk scores were calculated by adding each independent risk factor for recurrence-free survival (risk score ranged 0-12). Patients were divided by their score into low-risk (0-2), intermediate-risk (3-4), and highrisk (5-12) groups (Table 2). Recurrence-free survival was significantly shorter in patients with high-risk than with low-risk or intermediate-risk $(P<0.001)$ scores (Figure 2B). The number of patients who experienced visceral recurrence within 24 months was significantly higher in those with high-risk ( $n=65 / 158 ; 41 \%)$ versus low-risk $(n=3 / 133 ; 2.3 \%)$ or intermediate-risk scores $(n=13 / 136,9.6 \%)(P<0.001$; Figure $2 \mathrm{C})$. However, the number of patients with intravesical recurrence within 24 months was not significantly different among those with low-risk $(n=23 / 133 ; 17 \%)$, intermediate-risk $(n=$ $37 / 136,27 \%)$, and high-risk scores $(n=34 / 158 ; 22 \%)$ $(P=0.143)$ (Figure 2D). Based on this risk stratification, we developed a risk score-based protocol for oncological follow-up (Table 3) and evaluated per person cost of visceral recurrence detection. The estimated cost per detected recurrence was acceptable and estimated within $\$ 50,000$ for 5 years in all risk score groups except for the low-risk score patients at 12 months and the intermediaterisk score patients at 60 months (Figure 2E).

\section{Time course differences in patients with visceral recurrence $(n=109)$}

The risk score-based protocol effectively stratified patients with visceral recurrence compared to the pathology-based protocol. The pathology-based protocol showed higher incidence of visceral recurrence within 1 year in the high-risk (38\%) than in the normal-risk (9\%) and very high-risk (13\%) groups (Figure 3A). However, the risk score-based protocol showed higher incidence of visceral recurrence within 1 year in patients with high-risk $(50 \%)$ than in those with low-risk $(2 \%)$ and intermediaterisk (10\%) scores (Figure 3B). In total, 47 of 109 patients had symptomatic visceral recurrence. The number of patients with symptomatic recurrence in the normal-, high-, and very high-risk groups was 10 of 21 (48\%), 23 of $63(37 \%)$, and 14 of $25(56 \%)(P=0.225$; Figure 3C). The number of patients with symptomatic recurrence in 
the low-, intermediate-, and high-risk groups was 1 of 5 $(20 \%), 8$ of $22(36 \%)$, and 38 of $82(46 \%)$, respectively $(P=0.408$; Figure $3 \mathrm{C})$.

\section{Economic outcome differences between the pathology- and risk score-based protocols}

In the pathology-based protocol, median surveillance cost to detect 1 recurrence in 5 years was significantly different in the 3 groups $(P<0.001$, Kruskal-Wallis test; Figure 3D). Median estimated surveillance costs per detected visceral recurrence were $\$ 40,501, \$ 18,775$, and $\$ 8,622$ in the normal-, high-, and very high-risk groups. In the risk score-based protocol, median surveillance cost to detect 1 recurrence in 5 years was significantly different in the 3 groups $(P<0.001$, Kruskal-Wallis test, Figure 3D). The median estimated surveillance costs per detected visceral recurrence were $\$ 31,551, \$ 25,486$, and $\$ 5,140$ in the low-, intermediate-, and high-risk score groups, respectively. The risk score-based protocol led to a dramatic cost reduction compared to the pathologybased protocol. The total estimated 5-year surveillance cost was 2.2-fold higher with the pathology-based protocol
$(\$ 1,365,245)$ than with the risk score-based protocol $(\$ 617,315)$. The estimated cost difference was $\$ 747,929$ for 5 years (Figure 3E), a suggested 55\% cost reduction.

\section{DISCUSSION}

There is no strong evidence for an optimal followup protocol in UTUC patients after RNU [5, 6, 8], and no studies have been conducted to investigate the costeffectiveness of surveillance regimens after RNU. Although pathological stage is known to be a strong predictor of relapse, heterogeneity in UTUC patients prevents effective surveillance with a universal protocol for all patients. Our results showed that a pathology-based surveillance protocol successfully stratified the risk of visceral recurrence but potentially increased unnecessary testing in normal-risk ( $\leq$ pT2N0, LVI-, SM-) patients. Because pathological outcome and other clinical risk factors have a significant impact on tumor recurrence after RNU, we developed a risk score-based surveillance protocol including clinical risk factors and stratified the study patients into low-risk (0-2), intermediaterisk (3-4) and high-risk (5-12) score groups. As shown
A

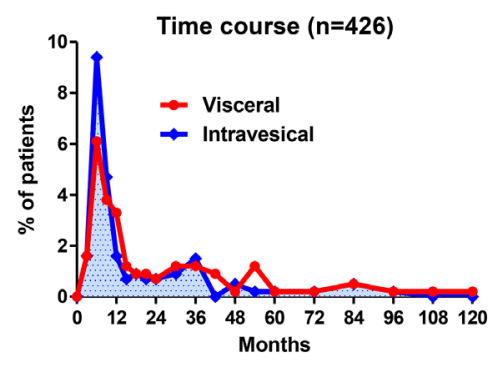

D Recurrence-free survival (intravesical)

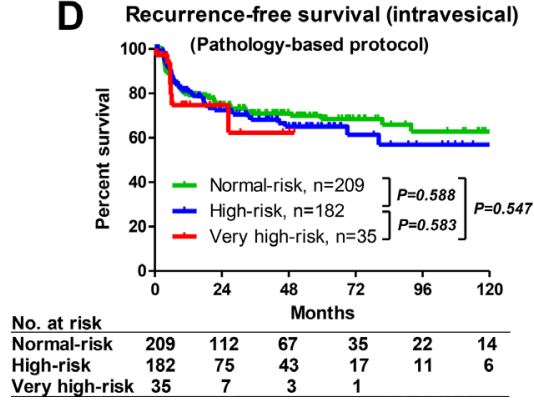

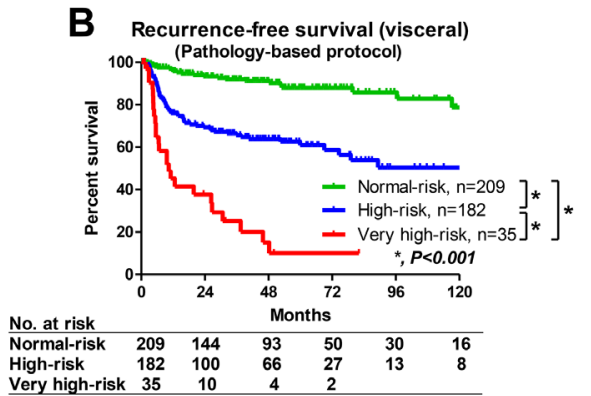
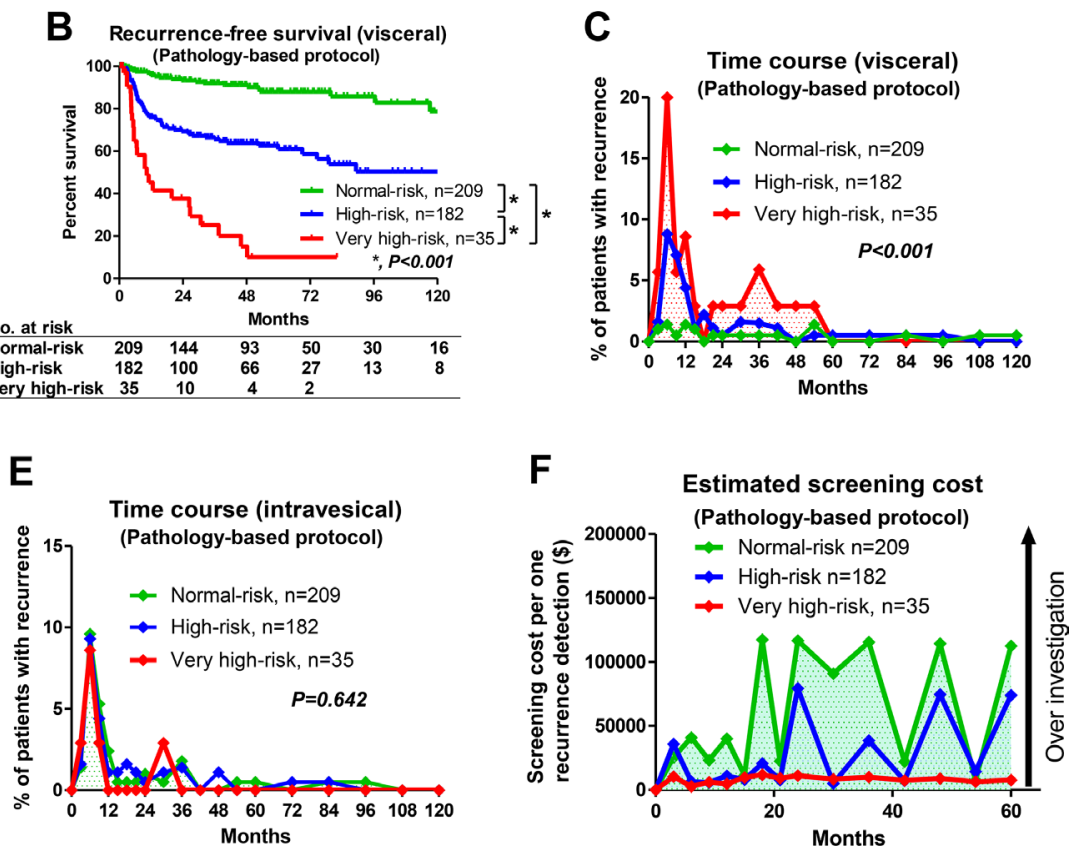

Figure 1: Oncological and economic outcomes of pathology-based surveillance protocols. (A) Time-course analysis of visceral and intravesical recurrence patterns. Number of patients with visceral and intravesical recurrences within 3 years after radical nephroureterectomy was 88/109 (81\%) and 96/113 (85\%), respectively. (B) Visceral recurrence-free survival in normal-risk $(\leq$ pT2N0, lymphovascular invasion- [LVI-], surgical margin- [SM-], high-risk [N0 with pT3 or LVI+]), and very high-risk (pT4, SM+, or N+) patients in the pathology-based protocol. There were significant differences in prognosis among the groups $(P<0.001)$. (C) Time-course analyses of visceral recurrence patterns of normal-, high- and very high-risk patients in the pathology-based protocol. There were significant differences in visceral recurrence patterns among the groups $(P<0.001)$. (D) Intravesical recurrence-free survival in normal-, high- and very high-risk patients in the pathology-based protocol. There were no significant differences in intravesical recurrence among the groups $(P=0.722)$. (E) Time-course analyses of intravesical recurrence patterns of normal-, high- and very high-risk patients in the pathology-based protocol. There were no significant differences in intravesical recurrence patterns among the groups $(P=0.642)$. (F) Estimated cost per detected recurrence in normal-, high- and very high-risk patients in the pathology-based protocol. Estimated costs for patients with normal risk were high $(>\$ 50,000)$ after 18 months. 
in Figure 2A, pathological outcomes and preoperative variables, including presence of stage 3 chronic kidney disease (CKD), hydronephrosis, and ureter/multiple tumor, had a significant impact on recurrence after RNU. The reason for the strong association between CKD and poor recurrence-free survival is not clear. Previous studies have suggested that kidney and urinary tract cancers are high risk for renal dysfunction due to tumor location (obstruction, and/or reduction of nephron mass) [16-18]. Our previous studies suggested that patients with stage 3 CKD had significantly higher risk for recurrence and cancer death compared to those without CKD [19-21]. In addition, UTUC has great potential for renal dysfunction during disease progression. Presence of hydronephrosis was an independent factor for recurrence in this study. Close relationship exists between presence of locally advanced UTUC, hydronephrosis, and renal impairment. Future studies to assess the underlying mechanisms of carcinogenesis and renal impairment are warranted.

Conversely, we could not address the effectiveness of a risk score-based protocol in intravesical recurrence. In univariate Cox regression analysis, previous/concomitant bladder cancer $(P=0.005)$ and stage $3 \mathrm{CKD}(P=0.008)$ were independent factors for intravesical recurrence (Supplementary Figure 1A). Although presence of hydronephrosis was not significant $(P=0.062)$, we included it as a risk score calculation due to its marginal influence on intravesical recurrence. Intravesical recurrence-free survival was significantly poor in patients with risk scores of 2-3 (high risk) compared to those with risk scores of 0-1 (low risk) (Supplementary Figure 1B; $P=0.001)$. However, the time course of intravesical recurrence between the low- and high-risk scores was not significantly different (Figure 2D). These results suggested that risk stratification for intravesical recurrence might not be useful. In addition, 91\% (103/119) of patients experienced intravesical recurrence within 36 months. Therefore, routine surveillance such as cystoscope and urine cytology every 3-6 months within 36 months might be optimal for detecting intravesical recurrence.

Our results suggested a potential benefit of this risk score-based protocol to reduce over investigation after RNU. However, the cost was substantial as the low relapse rate in patients with low-risk scores increased the
A

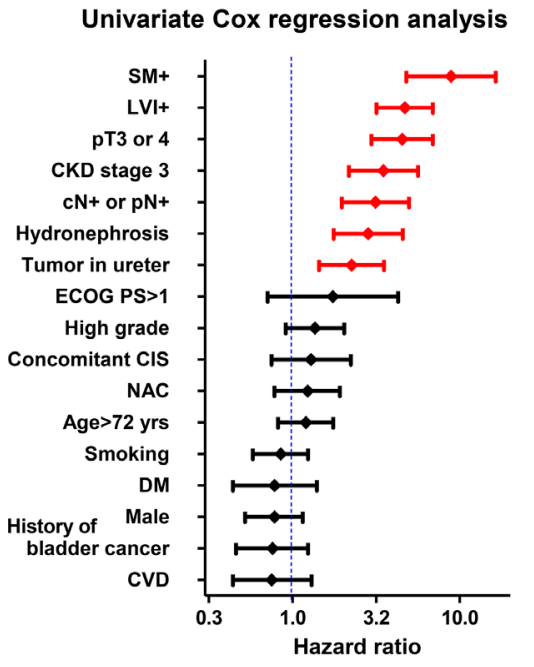

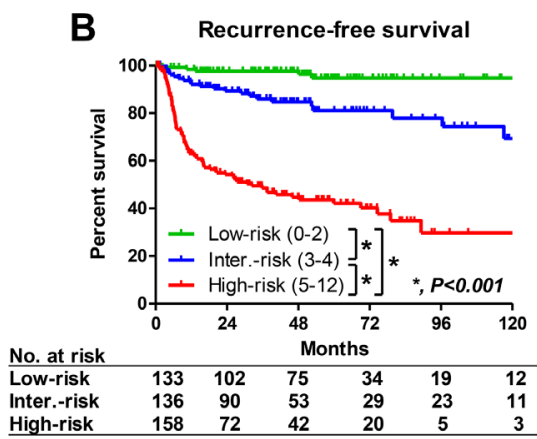

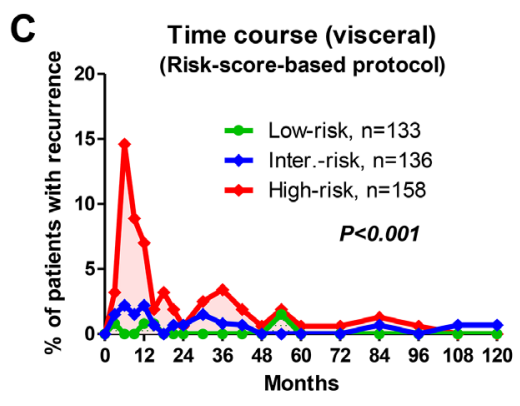

D

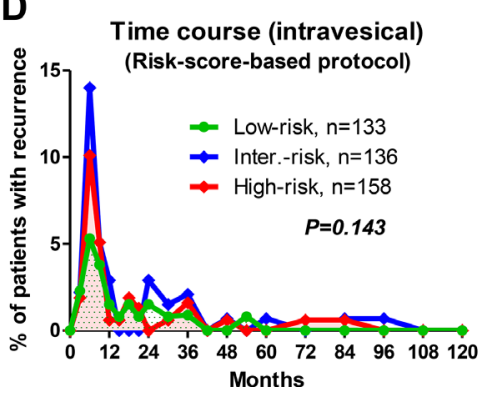

E

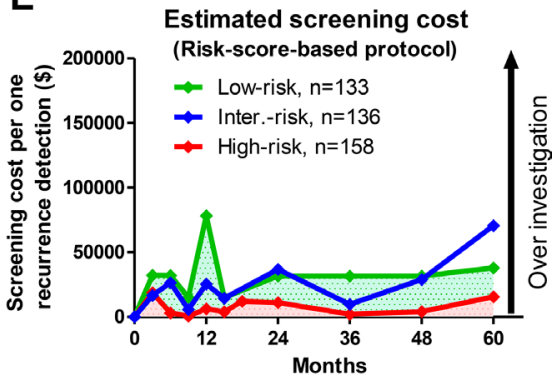

Figure 2: Oncological and economic outcomes of risk-score-based surveillance protocols. (A) Seven independent risk factors for visceral recurrence were selected using univariate analysis after radical nephroureterectomy (RNU). Risk scores were calculated using multivariate Cox regression analysis using tumor in ureter (score 1), presence of hydronephrosis (score 1), lymph node involvement (score 2), preoperative CKD (score 2), pT3-4 (score 2), LVI+ (score 2) and SM+ (score 2). (B) Visceral recurrence-free survival was shown for each risk score. Patients were stratified into 3 groups by low- (0-2), intermediate- (3-4) and high-risk scores (5-12), indicating the probability of relapse. There were significant differences in prognosis among the groups $(P<0.001)$. (C) Time-course analyses of recurrence in low-, intermediateand high-risk scores showed that the number of patients with visceral recurrence in the high-risk group was significantly higher compared to those in the low- and intermediate-risk groups. (D) Intravesical recurrence-free survival was shown for each risk score. There were no significant differences in intravesical recurrence among the groups $(P=0.143)$. (E) The estimated cost per detected recurrence was acceptable and estimated to be within $\$ 50,000$ for 5 years in all risk score groups except for the low-risk score patients at 12 months and the intermediaterisk score patients at 60 months. SM: surgical margin, LVI: lymphovascular invasion, CKD: chronic kidney disease, CVD: cardiovascular disease, HTN: hypertension, NAC: neoadjuvant chemotherapy, DM: diabetes mellitus. CIS: carcinoma in situ. 
Table 1: Background of patients

\begin{tabular}{|c|c|}
\hline$n$ & 426 \\
\hline Age (years) & $70 \pm 8.9$ \\
\hline Sex (Male), $n=$ & $290(68 \%)$ \\
\hline ECOG-PS $>1, n=$ & $10(2.3 \%)$ \\
\hline Hypertension, $n=$ & $185(43 \%)$ \\
\hline Diabetes Mellitus (DM), $n=$ & $70(16 \%)$ \\
\hline Cardiovascular disease (CVD), $n=$ & $75(18 \%)$ \\
\hline Smoking, $n=$ & $193(45 \%)$ \\
\hline eGFR before surgery $\left(\mathrm{ml} / \mathrm{min} / 1.73 \mathrm{~m}^{2}\right)$ & $58 \pm 18$ \\
\hline Hydronephrosis, $n=$ & $266(62 \%)$ \\
\hline Neoadjuvant chemotherapy (NAC), $n=$ & $102(24 \%)$ \\
\hline \multicolumn{2}{|l|}{ Clinical stage } \\
\hline$\geq \mathrm{cT} 3, n=$ & $229(54 \%)$ \\
\hline $\mathrm{cN}+, n=$ & $34(8.0 \%)$ \\
\hline \multicolumn{2}{|l|}{ Tumor location, $n=$} \\
\hline Renal pelvis & $166(39 \%)$ \\
\hline Ureter & $235(55 \%)$ \\
\hline Multiple & $25(5.9 \%)$ \\
\hline Laparoscopic surgery, $n=$ & $75(18 \%)$ \\
\hline Postoperative complications (G3 or higher), $n=$ & $14(3.3 \%)$ \\
\hline \multicolumn{2}{|l|}{ Pathological outcome, $n=$} \\
\hline$\geq \mathrm{pT} 3$ & $182(43 \%)$ \\
\hline $\mathrm{pN}+$ & $30(7.0 \%)$ \\
\hline High grade & $397(93 \%)$ \\
\hline Surgical margin (SM) positive & $14(3.3 \%)$ \\
\hline Lymphovascular invasion (LVI) positive & $127(30 \%)$ \\
\hline Adjuvant Chemotherapy, $n=$ & $44(10 \%)$ \\
\hline Median follow-up (Months) & 40 \\
\hline \multicolumn{2}{|l|}{ Disease recurrence, $n=$} \\
\hline Intravesical & $113(27 \%)$ \\
\hline Visceral & $109(26 \%)$ \\
\hline \multicolumn{2}{|l|}{ Deceased, $n=$} \\
\hline Cancer-specific & $80(19 \%)$ \\
\hline Any cause & $103(24 \%)$ \\
\hline
\end{tabular}

surveillance cost, highlighting the limitation of predicting relapse risk based exclusively on clinicopathological information. In addition, prediction of symptomatic recurrence is challenging. Several reports have suggested that presence of symptom at tumor recurrence is an independent predictor for poor prognosis, and detection of recurrence at the asymptomatic stage may improve prognosis [13, 22, 23]. However, our results showed that both pathology- and risk score-based stratification failed to separate the mode of recurrence. These results suggested that molecular biomarkers such as genomic subtypes in patients with symptomatic recurrence need to be investigated further to improve clinical management $[24,25]$

This study was limited by its retrospective design. First, we could not control the influence of all confounding factors, selection bias, and medical costs for oncological surveillance. Actual surveillance protocol might be not completely same in four different hospitals due to a retrospective manner. Selection biases exist for lymph node dissection and adjuvant chemotherapy administration in the present study. Recently, a phase III 
Table 2: Multivariate analysis for risk score calculation

\begin{tabular}{|c|c|c|c|c|}
\hline Factor & $P$ value & HR & $95 \% \mathrm{CI}$ & Risk score \\
\hline Tumor in ureter & $<0.001$ & 2.25 & $1.44-3.52$ & 1 \\
\hline Hydronephrosis & $<0.001$ & 2.83 & $1.76-4.56$ & 1 \\
\hline Lymph node involvement $(\mathrm{cN}+$ or $\mathrm{pN}+)$ & $<0.001$ & 3.13 & $1.97-4.97$ & 2 \\
\hline Preoperative CKD & $<0.001$ & 3.49 & $2.17-5.62$ & 2 \\
\hline pT3-4 & $<0.001$ & 4.52 & $2.96-6.89$ & 2 \\
\hline LVI+ & $<0.001$ & 4.68 & $3.18-6.89$ & 2 \\
\hline $\mathrm{SM}+$ & $<0.001$ & 8.85 & $4.78-16.4$ & 2 \\
\hline
\end{tabular}

CKD: chronic kidney disease, LVI: lymphovascular invasion, SM: surgical margin.

randomized trial of perioperative chemotherapy versus surveillance in UTUC (POUT trial) suggested benefit of adjuvant chemotherapy for disease free survival (DFS) for patients with histologically confirmed pT2-T4 N0-3 M0 UTUC [26]. Therefore, adjuvant chemotherapy (four cycles of gemcitabine-cisplatin or gemcitabine-carboplatin if estimated glomerular filtration rate [eGFR] $30-49 \mathrm{ml} /$ min) will be standard therapy shortly. Second, the risk scoring in the present study might not be optimal for risk stratification. The hazard ratio difference between lymph node involvement and surgical margin was more than two folds. However, when we stratified based on actual hazard ratio into scoring system, we found no big difference in risk stratification and outcomes. Therefore, we used simple scoring system in the present study. Third, pathological risk stratification of pT2 patients into the normal-risk group might not be suitable. It is reported that pT2 disease has a high risk of recurrence in Japanese population [27]. However, patients with pT3 disease has much higher risk for disease recurrence than those with pT2 disease. In the
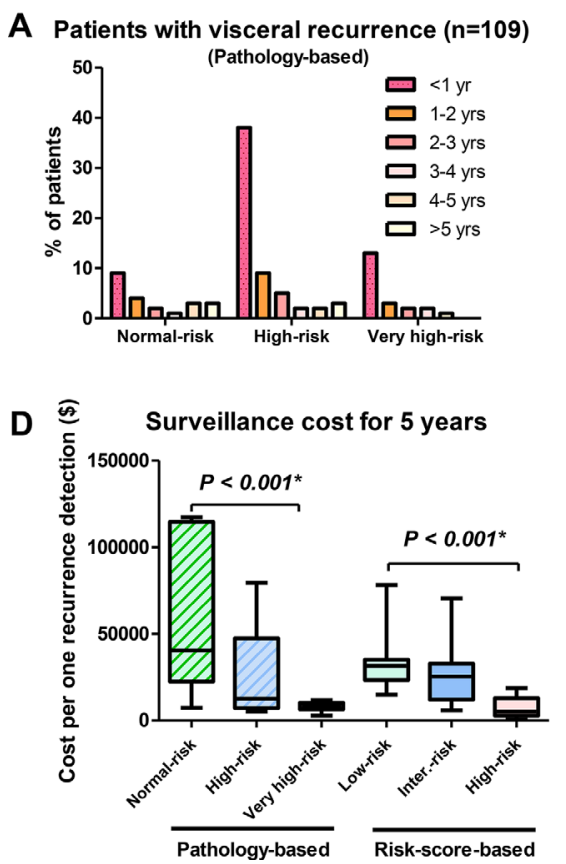

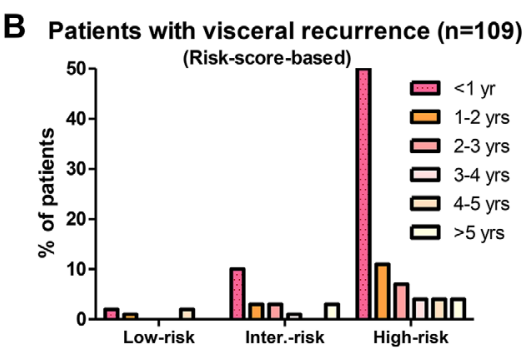

\section{E}

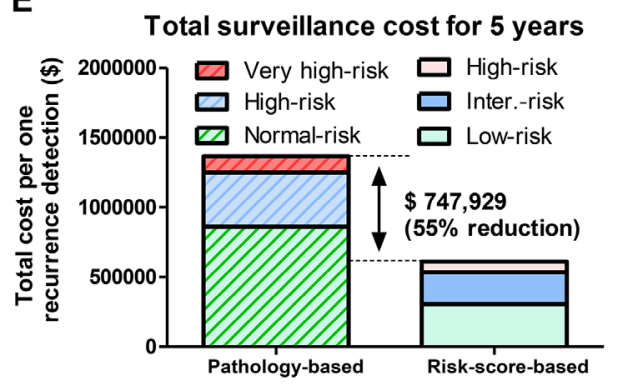

C Mode of visceral recurrence $(n=109)$
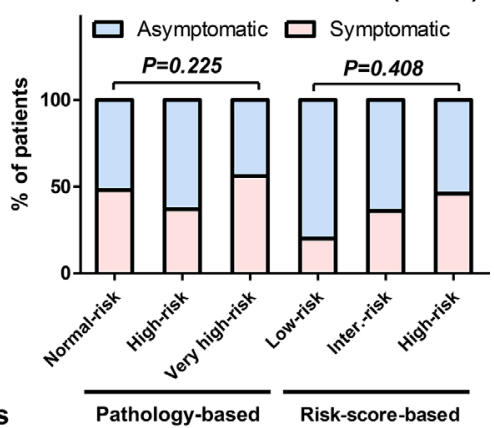

Figure 3: Time course and cost differences between the pathology-based and risk-score-based surveillance protocols. (A) The pathology-based protocol showed higher incidence of visceral recurrence within 1 year in the high-risk (38\%) than in the normalrisk (9\%) and very high-risk (13\%) groups. (B) The risk score-based protocol showed higher incidence of visceral recurrence within 1 year in patients with high-risk (50\%) than in those with low-risk (2\%) and intermediate-risk (10\%) scores. (C) The number of patients with symptomatic recurrence in the normal-, high-, and very high-risk groups was 10 of 21 (48\%), 23 of 63 (37\%), and 14 of 25 (56\%) $(P=0.225)$. The number of patients with symptomatic recurrence in the low-, intermediate-, and high-risk groups was 1 of $5(20 \%), 8$ of $22(36 \%)$, and 38 of $82(46 \%)$, respectively $(P=0.408)$. (D) In the pathology-based protocol, median surveillance cost to detect 1 recurrence in 5 years was significantly different in the 3 groups $(P<0.001$, Kruskal-Wallis test). In the risk score-based protocol, median surveillance cost to detect 1 recurrence in 5 years was significantly different in the 3 groups $(P<0.001$, Kruskal-Wallis test). (E) The total estimated 5-year surveillance cost was 2.2-fold higher with the pathology-based protocol $(\$ 1,365,245)$ than with the risk score-based protocol $(\$ 617,315)$. The estimated cost difference was $\$ 747,929$ for 5 years, a suggested $55 \%$ cost reduction. 
Table 3: Risk-score-based protocol

\begin{tabular}{|c|c|c|c|c|c|c|c|c|c|c|c|c|c|c|}
\hline \multirow{2}{*}{$\begin{array}{l}\text { Risk-score-based } \\
\text { Type of investigation }\end{array}$} & \multicolumn{14}{|c|}{ Months after RNU } \\
\hline & 3 & 6 & 9 & 12 & 15 & 18 & 21 & 24 & 30 & 36 & 42 & 48 & 54 & 60 \\
\hline $\begin{array}{l}\text { Basic exam (blood and serum test, ultrasound, } \\
\text { and chest X-ray) }\end{array}$ & $\bullet$ & $\bullet$ & & $\bullet$ & & $\bullet$ & & $\bullet$ & & $\bullet$ & & $\bullet$ & & $\bullet$ \\
\hline Urine analysis, cytology and cystoscope & $\bullet$ & $\bullet$ & $\bullet$ & $\bullet$ & $\bullet$ & $\bullet$ & & $\bullet$ & & $\bullet$ & & $\bullet$ & & $\bullet$ \\
\hline \multicolumn{15}{|l|}{ CT scan of chest/abdomen/pelvis } \\
\hline Low-risk (0-2) & & & & $\bullet$ & & & & & & & & & & $\bullet$ \\
\hline Intermediate-risk (3-4) & & $\bullet$ & & $\bullet$ & & & & $\bullet$ & & $\bullet$ & & $\bullet$ & & $\bullet$ \\
\hline High-risk (5-12) & • & - & • & - & $\bullet$ & $\bullet$ & & $\bullet$ & & $\bullet$ & & $\bullet$ & & $\bullet$ \\
\hline
\end{tabular}

RNU: radical nephroureterectomy.

present study, 2-year recurrence free survivals in pT1, pT2, pT3 and pT4 were $96 \%, 85 \%, 65 \%$, and $18 \%$, respectively. The difference in 2-year recurrence-free survival between pT1 and pT2 disease was smaller $(11 \% ; P=0.009)$ compared with between pT2 and pT3 $(20 \% ; P<0.001)$. In addition, $\mathrm{pT} 2$ patients who experienced disease recurrence had other significant risk factors such as $\mathrm{LVI}+/ \mathrm{pN}+$, stage $3 \mathrm{CKD}$, and preoperative hydronephrosis. There were significant differences in the number of pT2 patients between with and without recurrence in $\mathrm{LVI}+/ \mathrm{pN}+$ status ( $37 \%$ vs $16 \% ; P=0.034$ ), stage 3 CKD ( $95 \%$ vs. $52 \%$; $P<0.001)$, preoperative hydronephrosis (95\% vs. $58 \%$; $P=0.002)$. Therefore, pathological factor of pT2 alone was not strong predictor for disease recurrence. Based on these findings, we classified pT2 as a pathological low-risk group in the present study and developed risk-stratified protocol to overcome the limitation of pathological outcome. Fourth, the implication of this study may not be suitable for non-Japanese population. In addition, the risk score-based surveillance model might increase the number of patients not detected by surveillance. The estimated number of patients who potentially experienced the delay of detection and mean periods of delay were three patients $(2.3 \%)$ and 5.2 months in the risk-score-based low-risk group, nine patients $(6.6 \%)$ and 2.7 months in the riskscore-based intermediate-risk group, and 15 patients $(9.5 \%)$ and 1.1 months in the risk-score-based high-risk group, respectively. In total, the estimated number of patients with detection delay and mean periods of delay were 27 patients $(6.3 \%)$ and 1.7 months, respectively. Therefore, validation of both pathology-based and riskscore-based risk classification is needed. Despite these limitations, our study may give the impression that risk stratification in each nation is necessary to improve costeffectiveness in each medical system. A prospective study on the cost-effectiveness of follow up using a universal, standard, and easily applicable surveillance model is required to validate these results.
In conclusion, a risk score-based surveillance protocol has the potential to reduce over investigation during follow-up, making surveillance more cost-effective. Further study is needed to determine the impact of risk stratification on the cost-effectiveness of oncological follow-up after RNU.

\section{MATERIALS AND METHODS}

\section{Patient selection and variables}

Between May 1995 and February 2017, 426 consecutive adults underwent RNU at Hirosaki University Hospital, Aomori Rosai Hospital, Mutsu General Hospital, and Aomori Prefectural Central Hospital. Inclusion criteria were UTUC patients without distant metastasis who underwent RNU. Exclusion criteria were lack of sufficient variables recorded in the chart including clinicopathological and oncological outcomes. All patients were treated with RNU with or without perioperative systemic chemotherapy. Based on pathological outcome after RNU, all patients were stratified for oncological follow-up to normal-risk ( $\leq$ pT2N0), high-risk (N0 with pT3 or LVI + ) and very high-risk (pT4, SM+, or lymph node involvement $[\mathrm{cN}+$ or $\mathrm{pN}+]$ ) groups. The variables analyzed were age, sex, Eastern Cooperative Oncology Group Performance Status (ECOG PS), smoking, clinical stage, preoperative CKD (eGFR $<60 \mathrm{~mL} / \mathrm{min} / 1.73 \mathrm{~m}^{2}$ ) [28], hydronephrosis, history of hypertension (HTN), cardiovascular disease (CVD), and diabetes mellitus (DM). Tumor stage and grade were defined by the 2009 TNM classification [29]. Postoperative complications were evaluated by the Clavien-Dindo classification [30]. This retrospective study was approved by the Ethics Committee of the Hirosaki University School of Medicine (authorization numbers 2017-089) including all hospitals. 


\begin{tabular}{|c|c|c|c|c|c|c|c|c|c|c|c|c|c|c|}
\hline \multirow{2}{*}{$\begin{array}{l}\text { Pathology-based } \\
\text { Type of investigation }\end{array}$} & \multicolumn{14}{|c|}{ Months after RNU } \\
\hline & 3 & 6 & 9 & 12 & 15 & 18 & 21 & 24 & 30 & 36 & 42 & 48 & 54 & 60 \\
\hline $\begin{array}{l}\text { Basic exam (blood and serum test, } \\
\text { ultrasound, and/or chest X-ray) }\end{array}$ & • & $\bullet$ & & $\bullet$ & & $\bullet$ & & $\bullet$ & & $\bullet$ & & $\bullet$ & & $\bullet$ \\
\hline Urine analysis, cytology and cystoscope & $\bullet$ & $\bullet$ & $\bullet$ & • & • & • & • & • & • & • & • & • & • & $\bullet$ \\
\hline \multicolumn{15}{|l|}{ CT scan of chest/abdomen/pelvis } \\
\hline Normal-risk ( $\leq$ pT2N0, LVI-, SM-) & & $\bullet$ & & $\bullet$ & & $\bullet$ & & $\bullet$ & & $\bullet$ & & $\bullet$ & & $\bullet$ \\
\hline High-risk (N0 with pT3, or LVI+) & $\bullet$ & $\bullet$ & $\bullet$ & $\bullet$ & & $\bullet$ & & $\bullet$ & & $\bullet$ & & $\bullet$ & & $\bullet$ \\
\hline Very high-risk (pT4, $\mathrm{SM}+$, or $\mathrm{N}+$ ) & $\bullet$ & $\bullet$ & $\bullet$ & $\bullet$ & $\bullet$ & $\bullet$ & $\bullet$ & • & $\bullet$ & • & $\bullet$ & • & • & • \\
\hline
\end{tabular}

RNU: radical nephroureterectomy, LVI: lymphovascular invasion, SM: surgical margin.

\section{Surgical procedure}

Open or laparoscopic nephroureterectomy, which includes the removal of kidney, ureter, and ipsilateral bladder cuff, was performed $[1,31]$. The distal ureter was managed by the extravesical approach. Because lymph node dissection for UTUC is still controversial and appropriate dissection area has never been established yet, we underwent regional lymph node dissection or sampling was performed depending on tumor stage. When an obvious nodule existed, regional lymph node dissection was performed. When patients had $\geq \mathrm{cT} 2$ disease without lymph node involvement, lymph node sampling was performed in selected patients. We did not use early (within $48 \mathrm{~h}$ ) intravesical chemotherapy after RNU.

\section{Systemic chemotherapy}

We have performed 2-4 courses of platinum-based neoadjuvant chemotherapy (NAC) for the treatment of locally advanced UTUC (cT3-4 and/or $\mathrm{cN}+$ ) in selected patients. Regimens were selected based on guidelines regarding eligibility for the proper use of cisplatin [32] and the patient's overall status. Adjuvant chemotherapy was administered in selected patients (high- or very high-risk patients with suitable general health status). Salvage chemotherapy after recurrence consisted of a platinum-based combination regimen. Regimens were selected based on residual renal function and overall status.

\section{Surveillance protocol}

Oncological follow-up after RNU was performed following the several guidelines and previously published pathology protocols $[14,33]$. The pathology-based protocol is shown in Table 4. Evaluations by bone scans or brain imaging were performed when clinically indicated.
Visceral organ recurrence included metastasis to the liver, lungs, adrenal glands, and other intra-abdominal organs.

\section{Outcome measurements}

Recurrence-free survival, time to recurrence, and estimated cost per recurrence detected by the pathologybased protocol were recorded for the normal-, high-, and very high-risk groups. To estimate cost-benefit, we calculated the medical cost of follow-up to detect 1 recurrence ([surveillance cost in a follow-up period] / [number of patients with recurrence]) using an exchange rate of 100 yen to the U.S. dollar. Estimated medical costs were $\$ 350$ for computed tomography (CT), $\$ 70$ for blood testing, $\$ 53$ for ultrasonography, and $\$ 25$ for urine cytology. The cost of prescribing medications, and doctor fees were not included in the analysis. A total examination fee was calculated as a total patient's fee (10-30\%) and national insurance coverage $(70-90 \%)$ in Japan. To compare cost-effectiveness, we developed a novel risk score-based protocol using multivariate Cox proportional hazards regression. Patient risk scores were calculated by summing the number of independent risks suggested in the multivariate analysis, and the patients were stratified as low (0-2), intermediate (3-4), and high (5-12) risk. Next, we developed our ad-hoc surveillance protocol that enhances cost-effectiveness without increasing the number of patients who are not detected by oncological screening based on the frequency of recurrences. The estimated cost per detected recurrence in the pathology-based and risk score-based protocols was compared.

\section{Statistical analysis}

Statistical analysis was performed using SPSS version 24.0 (IBM Corp. Released 2016. IBM SPSS Statistics for Windows, Version 24.0. Armonk, NY, USA: IBM Corp.) and GraphPad Prism 5.03 (GraphPad 
Software, San Diego, CA, USA). Categorical variables were compared using Fisher exact test or the chi-square test. Differences between groups were compared using $t$ tests or Mann-Whitney $U$ test. The Kruskal-Wallis test was used to compare medians among the 3 groups. All tests were 2 -sided, and a $P$ value $<0.05$ was considered statistically significant. Recurrence-free survival in patients with recurrence stratified by risk criteria was estimated using the Kaplan-Meier method and compared by the log-rank test. Uni- and multivariate Cox proportional hazards regression were used to identify factors independently associated with recurrence-free survival; hazard ratios (HRs) with 95\% confidence intervals (CIs) were calculated after simultaneously controlling for potential confounders including patient demographic and clinicopathological variables.

\section{Ethical standards}

This study was performed in accordance with the ethical standards of the Declaration of Helsinki and approved by an ethics review board of Hirosaki University School of Medicine (authorization numbers 2017-089).

\section{Informed consent}

For this type of study, formal written consent is not required. Pursuant to the provisions of the ethics committee and the ethic guideline in Japan, written consent was not required in exchange for public disclosure of study information in the case of retrospective and/or observational study using a material such as the existing documentation. The study information was open for the public consumption at http://www.med.hirosaki-u. ac.jp/ uro/html/IRB/IRBdoc.html.

\section{Abbreviations}

UTUC: upper tract urothelial carcinoma, RNU: radical nephroureterectomy, LVI: lymphovascular invasion, SM: surgical margin, ECOG PS: Eastern Cooperative Oncology Group Performance Status, HTN: hypertension, CVD: cardiovascular disease, DM: diabetes mellitus, eGFR: estimated glomerular filtration rate, CKD: chronic kidney disease, NAC: neoadjuvant chemotherapy, HR: hazard ratio, 95\% CI: 95\% confidence interval.

\section{Author contributions}

Conception and design: Shingo Hatakeyama, Chikara Ohyama. Acquisition of data: Masaki Momota, Shingo Hatakeyama, Hayato Yamamoto, Hiromichi Iwamura, Takahiro Yoneyama, Yasuhiro Hashimoto, Takuya Koie, Toshiaki Kawaguchi. Analysis and interpretation of data: Shingo Hatakeyama, Masaki Momota. Drafting of the manuscript: Shingo Hatakeyama, Masaki Momota. Critical revision of the manuscript: Yuki Tobisawa, Tohru Yoneyama. Statistical analysis: Shingo Hatakeyama, Tohru Yoneyama. Obtaining funding: Shingo Hatakeyama, Takuya Koie, Chikara Ohyama. Administrative, technical, or material support: Yuki Tobisawa, Tohru Yoneyama, Chikara Ohyama.

\section{ACKNOWLEDGMENTS}

We thank Hiroyuki Ito, Kazuaki Yoshikawa, Atsushi Sasaki, Naoki Fujita, Ken Fukushi, Jotaro Mikami, Toshikazu Tanaka, Takuma Narita, Itsuto Hamano, Yuka Kubota, Yuki Fujita, Yukie Nishizawa, and Satomi Sakamoto for their invaluable help with data collection. The authors would like to thank Enago (www.enago.jp) for the English language review.

\section{CONFLICTS OF INTEREST}

The authors declare no conflicts of interest.

\section{FUNDING}

This work was supported by a Grant-in-Aid for Scientific Research (No. 15H02563, 15K15579, 17K11118, $17 \mathrm{~K} 11119,17 \mathrm{~K} 16768,17 \mathrm{~K} 16770$, and 17K16771) from the Japan Society for the Promotion of Science.

\section{REFERENCES}

1. Miyazaki J, Nishiyama H, Fujimoto H, Ohyama C, Koie T, Hinotsu S, Kikuchi E, Sakura M, Inokuchi J, Hara T. Laparoscopic Versus Open Nephroureterectomy in MuscleInvasive Upper Tract Urothelial Carcinoma: Subanalysis of the Multi-Institutional National Database of the Japanese Urological Association. J Endourol. 2016; 30:520-5. https:// doi.org/10.1089/end.2015.0757.

2. Roupret M, Babjuk M, Comperat E, Zigeuner R, Sylvester RJ, Burger M, Cowan NC, Bohle A, Van Rhijn BW, Kaasinen E, Palou J, Shariat SF. European Association of Urology Guidelines on Upper Urinary Tract Urothelial Cell Carcinoma: 2015 Update. Eur Urol. 2015; 68:868-79. https://doi.org/10.1016/j.eururo.2015.06.044.

3. Lughezzani G, Burger M, Margulis V, Matin SF, Novara G, Roupret M, Shariat SF, Wood CG, Zigeuner R. Prognostic factors in upper urinary tract urothelial carcinomas: a comprehensive review of the current literature. Eur Urol. 2012; 62:100-14. https://doi.org/10.1016/j. eururo.2012.02.030.

4. Mathieu R, Bensalah K, Lucca I, Mbeutcha A, Roupret M, Shariat SF. Upper urinary tract disease: what we know today and unmet needs. Transl Androl Urol. 2015; 4:26172. https://doi.org/10.3978/j.issn.2223-4683.2015.05.01. 
5. Oya M, Kikuchi E. Evidenced-based clinical practice guideline for upper tract urothelial carcinoma (summary-Japanese Urological Association, 2014 edition). Int J Urol. 2015; 22:3-13. https://doi.org/10.1111/iju.12630.

6. Seisen T, Colin P, Hupertan V, Yates DR, Xylinas E, Nison L, Cussenot O, Neuzillet Y, Bensalah K, Novara G, Montorsi F, Zigeuner R, Remzi M, et al. Postoperative nomogram to predict cancer-specific survival after radical nephroureterectomy in patients with localised and/or locally advanced upper tract urothelial carcinoma without metastasis. BJU Int. 2014; 114:733-40. https://doi. org/10.1111/bju.12631.

7. Hosogoe S, Hatakeyama S, Kusaka A, Hamano I, Iwamura H, Fujita N, Yamamoto H, Tobisawa Y, Yoneyama T, Yoneyama T, Hashimoto Y, Koie T, Ohyama C. Platinumbased Neoadjuvant Chemotherapy Improves Oncological Outcomes in Patients with Locally Advanced Upper Tract Urothelial Carcinoma. Eur Urol Focus. 2017. https://doi. org/10.1016/j.euf.2017.03.013.

8. Petrelli F, Yasser Hussein MI, Vavassori I, Barni S. Prognostic Factors of Overall Survival in Upper Urinary Tract Carcinoma: A Systematic Review and Meta-analysis. Urology. 2017; 100:9-15. https://doi.org/10.1016/j.urology.2016.07.036.

9. Kubota Y, Hatakeyama S, Tanaka T, Fujita N, Iwamura H, Mikami J, Yamamoto H, Tobisawa Y, Yoneyama T, Yoneyama T, Hashimoto Y, Koie T, Ito H, et al. Oncological outcomes of neoadjuvant chemotherapy in patients with locally advanced upper tract urothelial carcinoma: a multicenter study. Oncotarget. 2017; 8:101500-8. https:// doi.org/10.18632/oncotarget.21551.

10. Horiguchi H, Hatakeyama S, Anan G, Kubota Y, Kodama H, Momota M, Kido K, Yamamoto H, Tobisawa Y, Yoneyama T, Yoneyama T, Hashimoto Y, Koie T, et al. Detecting asymptomatic recurrence after radical nephroureterectomy contributes to better prognosis in patients with upper urinary tract urothelial carcinoma. Oncotarget. 2018; 9:8746-55. https://doi.org/10.18632/oncotarget.23982.

11. Witjes JA, Compérat E, Cowan NC, De Santis M, Gakis G, Lebret T, Ribal MJ, Van der Heijden AG, Sherif A; European Association of Urology. Treatment of muscleinvasive and metastatic bladder cancer: update of the EAU guidelines. Eur Urol. 2011; 59:1009-18. https://doi. org/10.1016/j.eururo.2011.03.023.

12. Volkmer BG, Schnoeller T, Kuefer R, Gust K, Finter F, Hautmann RE. Upper urinary tract recurrence after radical cystectomy for bladder cancer--who is at risk? J Urol. 2009; 182:2632-7. https://doi.org/10.1016/j.juro.2009.08.046.

13. Giannarini G, Kessler TM, Thoeny HC, Nguyen DP, Meissner C, Studer UE. Do patients benefit from routine follow-up to detect recurrences after radical cystectomy and ileal orthotopic bladder substitution? Eur Urol. 2010; 58:486-94. https://doi.org/10.1016/j.eururo.2010.05.041.

14. Yafi FA, Aprikian AG, Fradet Y, Chin JL, Izawa J, Rendon R, Estey E, Fairey A, Cagiannos I, Lacombe L, Lattouf JB, Bell D, Saad F, et al. Surveillance guidelines based on recurrence patterns after radical cystectomy for bladder cancer: the Canadian Bladder Cancer Network experience. BJU Int. 2012; 110:1317-23. https://doi.org/10.1111/j.1464410X.2012.11133.x.

15. Kusaka A, Hatakeyama S, Hosogoe S, Hamano I, Iwamura H, Fujita N, Fukushi K, Narita T, Hagiwara K, Yamamoto H, Tobisawa Y, Yoneyama T, Yoneyama T, et al. Riskstratified surveillance and cost effectiveness of follow-up after radical cystectomy in patients with muscle-invasive bladder cancer. Oncotarget. 2017; 8:65492-505. https://doi. org/10.18632/oncotarget.19043.

16. Na SY, Sung JY, Chang JH, Kim S, Lee HH, Park YH, Chung W, Oh KH, Jung JY. Chronic kidney disease in cancer patients: an independent predictor of cancer-specific mortality. Am J Nephrol. 2011; 33:121-30. https://doi. org/10.1159/000323740.

17. Yang Y, Li HY, Zhou Q, Peng ZW, An X, Li W, Xiong LP, Yu XQ, Jiang WQ, Mao HP. Renal Function and AllCause Mortality Risk Among Cancer Patients. Medicine (Baltimore). 2016; 95:e3728. https://doi.org/10.1097/ md.0000000000003728.

18. Wong G, Hayen A, Chapman JR, Webster AC, Wang JJ, Mitchell P, Craig JC. Association of CKD and cancer risk in older people. J Am Soc Nephrol. 2009; 20:1341-50. https:// doi.org/10.1681/asn.2008090998.

19. Hamano I, Hatakeyama S, Iwamura H, Fujita N, Fukushi K, Narita T, Hagiwara K, Kusaka A, Hosogoe S, Yamamoto H, Tobisawa Y, Yoneyama T, Yoneyama T, et al. Preoperative chronic kidney disease predicts poor oncological outcomes after radical cystectomy in patients with muscle-invasive bladder cancer. Oncotarget. 2017; 8:61404-14. https://doi. org/10.18632/oncotarget.18248.

20. Kodama H, Hatakeyama S, Fujita N, Iwamura H, Anan G, Fukushi K, Narita T, Tanaka T, Kubota Y, Horiguchi H, Momota M, Kido K, Matsumoto T, et al. Preoperative chronic kidney disease predicts poor oncological outcomes after radical nephroureterectomy in patients with upper urinary tract urothelial carcinoma. Oncotarget. 2017; 8:83183-94. https://doi.org/10.18632/oncotarget.20554.

21. Momota M, Hatakeyama S, Tokui N, Sato T, Yamamoto H, Tobisawa Y, Yoneyama T, Yoneyama T, Hashimoto Y, Koie T, Narita S, Kawaguchi T, Ohyama C. The Impact of Preoperative Severe Renal Insufficiency on Poor Postsurgical Oncological Prognosis in Patients with Urothelial Carcinoma. Eur Urol Focus. 2018 Mar 13. https:// doi.org/10.1016/j.euf.2018.03.003. [Epub ahead of print].

22. Alimi Q, Verhoest G, Kammerer-Jacquet SF, Mathieu R, Rioux-Leclercq N, Manunta A, Laguerre B, Guille F, Bensalah K, Peyronnet B. Role of routine computed tomography scan in the oncological follow up of patients treated by radical cystectomy for bladder cancer. Int J Urol. 2016; 23:840-6. https://doi.org/10.1111/iju.13164.

23. Kusaka A, Hatakeyama S, Hosogoe S, Hamano I, Iwamura H, Fujita N, Fukushi K, Narita T, Yamamoto H, Tobisawa Y, Yoneyama T, Yoneyama T, Hashimoto Y, et al. Detecting 
asymptomatic recurrence after radical cystectomy contributes to better prognosis in patients with muscleinvasive bladder cancer. Med Oncol. 2017; 34:90. https:// doi.org/10.1007/s12032-017-0955-9.

24. Choi W, Porten S, Kim S, Willis D, Plimack ER, HoffmanCensits J, Roth B, Cheng T, Tran M, Lee IL, Melquist J, Bondaruk J, Majewski T, et al. Identification of distinct basal and luminal subtypes of muscle-invasive bladder cancer with different sensitivities to frontline chemotherapy. Cancer Cell. 2014; 25:152-65. https://doi.org/10.1016/j. ccr.2014.01.009.

25. McConkey DJ, Choi W, Dinney CP. Genetic subtypes of invasive bladder cancer. Curr Opin Urol. 2015; 25:449-58. https://doi.org/10.1097/mou.0000000000000200.

26. Birtle AJ, Chester JD, Jones RJ, Johnson M, Hill M, Bryan RT, Catto J, Donovan J, French A, Harris C, Keeley F, Kockelbergh R, Powles T, et al. Results of POUT: A phase III randomised trial of perioperative chemotherapy versus surveillance in upper tract urothelial cancer (UTUC). Journal of Clinical Oncology. 2018; 36:407. https://doi. org/10.1200/JCO.2018.36.6_suppl.407.

27. Inokuchi $J$, Eto $M$, Hara $T$, Fujimoto $H$, Nishiyama $H$, Miyazaki J, Kikuchi E, Hinotsu S, Koie T, Ohyama C. Impact of lymph node dissection on clinical outcomes during nephroureterectomy in patients with clinically nodenegative upper urinary tract urothelial cancer: subanalysis of a multi-institutional nationwide case series of the Japanese Urological Association. Jpn J Clin Oncol. 2017; 47:652-9. https://doi.org/10.1093/jjco/hyx051.

28. Matsuo S, Imai E, Horio M, Yasuda Y, Tomita K, Nitta K, Yamagata K, Tomino Y, Yokoyama H, Hishida A. Revised equations for estimated GFR from serum creatinine in Japan. Am J Kidney Dis. 2009; 53:982-92. https://doi. org/10.1053/j.ajkd.2008.12.034.
29. Sobin LH, Gospodarowicz MK, Wittekind C, International Union Against Cancer. TNM classification of malignant tumours. (Chi chester, West Sussex, UK: Hoboken, NJ: Wiley-Blackwell). 2009.

30. Clavien PA, Barkun J, de Oliveira ML, Vauthey JN, Dindo D, Schulick RD, de Santibanes E, Pekolj J, Slankamenac K, Bassi C, Graf R, Vonlanthen R, Padbury R, et al. The Clavien-Dindo classification of surgical complications: five-year experience. Ann Surg. 2009; 250:187-96. https:// doi.org/10.1097/SLA.0b013e3181b13ca2.

31. Kido K, Hatakeyama S, Fujita N, Yamamoto H, Tobisawa Y, Yoneyama T, Yoneyama T, Hashimoto Y, Koie T, Iwabuchi I, Ogasawara M, Kawaguchi T, Ohyama C. Oncologic outcomes for open and laparoscopic radical nephroureterectomy in patients with upper tract urothelial carcinoma. Int J Clin Oncol. 2018 Feb 12. https://doi. org/10.1007/s10147-018-1248-9. [Epub ahead of print].

32. Galsky MD, Hahn NM, Rosenberg J, Sonpavde G, Hutson T, Oh WK, Dreicer R, Vogelzang N, Sternberg C, Bajorin DF, Bellmunt J. A consensus definition of patients with metastatic urothelial carcinoma who are unfit for cisplatinbased chemotherapy. Lancet Oncol. 2011; 12:211-4. https:// doi.org/10.1016/S1470-2045(10)70275-8.

33. Stewart-Merrill SB, Boorjian SA, Thompson RH, Psutka SP, Cheville JC, Thapa P, Bergstrahl EJ, Tollefson MK, Frank I. Evaluation of current surveillance guidelines following radical cystectomy and proposal of a novel riskbased approach. Urol Oncol. 2015; 33:339.e1-8. https://doi. org/10.1016/j.urolonc.2015.04.017. 